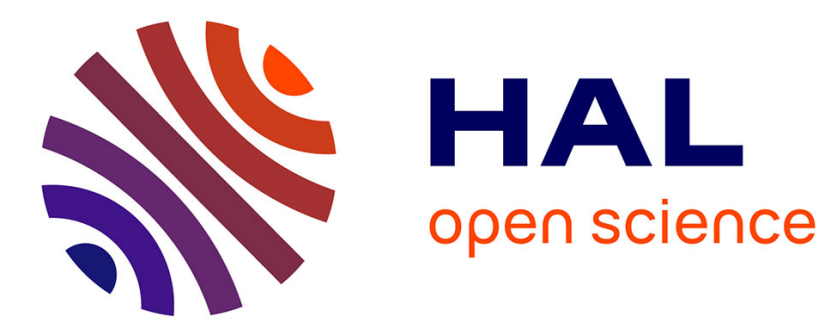

\title{
Impact of a Medium-Size Wave Farm on Grids of Different Strength Levels
}

Anne Blavette, Dara O’Sullivan, Ray Alcorn, Tony Lewis, Michael Egan

\section{To cite this version:}

Anne Blavette, Dara O'Sullivan, Ray Alcorn, Tony Lewis, Michael Egan. Impact of a Medium-Size Wave Farm on Grids of Different Strength Levels. IEEE Transactions on Power Systems, 2014, 29 (2), pp.917-923. 10.1109/TPWRS.2013.2284513 . hal-01266006

\section{HAL Id: hal-01266006 https://hal.science/hal-01266006}

Submitted on 26 Jan 2018

HAL is a multi-disciplinary open access archive for the deposit and dissemination of scientific research documents, whether they are published or not. The documents may come from teaching and research institutions in France or abroad, or from public or private research centers.
L'archive ouverte pluridisciplinaire HAL, est destinée au dépôt et à la diffusion de documents scientifiques de niveau recherche, publiés ou non, émanant des établissements d'enseignement et de recherche français ou étrangers, des laboratoires publics ou privés. 


\title{
Impact of a Medium-Size Wave Farm on Grids of Different Strength Levels
}

\author{
Anne Blavette*, Dara L. O’Sullivan*, Ray Alcorn*, Tony W. Lewis*, Michael G. Egan ${ }^{\dagger}$ \\ ${ }^{*}$ Beaufort Research-HMRC, University College Cork, Ireland \\ anne.blavette@umail.ucc.ie \\ ${ }^{\dagger}$ Electrical Engineering Department, University College Cork, Ireland
}

\begin{abstract}
Power fluctuations generated by most oscillating wave energy converters may have a negative impact on the power quality of the local grid to which the wave farms will be connected. Hence, assessing their impact is an important step in the selection process of a suitable deployment location. However, site-specific grid impact assessment studies are relatively time-consuming and require a high level of detail on the local network. Both of these constraints mean that grid impact studies are usually not performed in the preliminary stages of the site selection process, despite the extremely negative consequences resulting from poor power quality. This paper details a comprehensive study based on a relatively typical wave farm design connected to networks of different strength levels. The study was performed using experimental electrical power time series of an oscillating water column (OWC) device generated under the framework of the European FP7 project "CORES". Simulations were performed using DIgSILENT power system simulator "PowerFactory".
\end{abstract}

Index Terms-Wave energy, flicker, short-circuit ratio

\section{INTRODUCTION}

The rapid fluctuations of power sources such as wave and wind farms may deteriorate significantly the power quality in the local networks to which they are connected. In particular, the voltage fluctuations they induce may lead to an excessive level of flicker. However, radical divergences exist between wind and wave farms which prevent the conclusions of previous works focusing on wind turbines from being directly applicable to wave energy converters. Firstly, due to the nature of wave power, wave farms are expected to induce voltage fluctuations in a much lower frequency range than wind farms [1]. This decreases inherently the corresponding flicker level, as illustrated on the curve shown in Fig. 1 which represents the average perceptibility of individuals to luminous intensity fluctuations.

However, although the voltage fluctuations are generated in a lower frequency range, their amplitude is much higher as the power output of a wave energy converter may fluctuate between

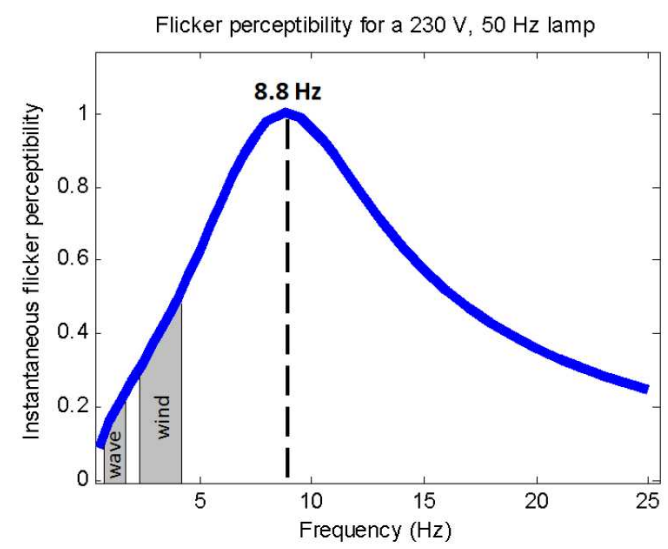

Figure 1: Flicker perceptibility curve as defined in IEC standard 61000-4-15 [2]

zero and a peak value on the short timescale of seconds, thus increasing the corresponding flicker level. In addition, the architecture of wave farms itself represents a source of divergence compared to wind farms: unlike onshore wind farms, mediumsize wave farms include relatively long submarine cables in the range of few tens of kilometers whose reactive power generation/consumption has a strong influence on the voltage of the local network, especially on highly inductive networks (i.e. whose impedance angle $\Psi_{k}=\arctan (\mathrm{X} / \mathrm{R})$ is sufficiently great). However, unlike large offshore wind farms which also include long submarine cables, the number of devices composing the medium-size wave farms to be deployed in the near future is much smaller, which limits the beneficial effect of aggregation on the farm's power output. Consequently, the grid connection of medium-size wave farms may induce a significant level of flicker. Hence, their impact must be carefully assessed before they are allowed grid connection. However, although the experience of the wind energy industry constitutes a relevant starting point with respect to wave energy, the estimation of the level of flicker generated by a wave farm requires specific studies. 
Grid impact assessment studies are typically performed on a site-by-site basis which is relatively time-consuming and thus prevents usually detailed analyses, such as flicker analyses, from being included in the selection process of a suitable site location. In addition, although several studies have already investigated the potential grid impact generated by a small to medium-size wave farm, the ratio of the local grid's short-circuit level to the farm's maximum power was in all cases either very low or very high [3], [6]. In the first case, the considered grids were significantly affected by the injection of fluctuating power, whereas in the second case, grids were not affected at all, as expected. Flicker level was also investigated regardless of the grid strength level but only in the context of a small farm [7]. Hence, it appears that the knowledge on the grid impact of a mediumsize wave farm was somewhat limited to sites presenting extreme characteristics in terms of shortcircuit ratio. Hence, in order to provide information on the flicker level generated by a medium-size wave farm on different types of grids, the generic study presented in this article has focused on the impact of a $20 \mathrm{MW}$-rated wave farm connected to grids presenting a wide range of strength levels, in terms of both short-circuit ratio and impedance angle $\Psi_{k}$, as detailed in Section II-C. Simulations were performed using DIgSILENT power system simulator "PowerFactory".

\section{MODELling}

\section{A. Experimental data}

Input data in the form of generated electrical power output time series was provided as an outcome of the European FP7 project CORES standing for "Components for Ocean Renewable Energy Systems" [8]. The project itself was based on a floating quarter-scale oscillating water colum (OWC) prototype deployed in Galway Bay, Ireland from March to May 2011. The device was connected to a small on-board islanded grid shown in Fig. 2, independent of the national electrical network. The on-board grid consisted of a generator powered by waves which supplied a battery, of a load intended to dump the excess of energy in the case where the battery were full, and of a backup diesel generator whose role was to supply the on-board network should the battery be empty. The project has generated a considerable amount of time series data on a number of parameters, including electrical parameters at a high temporal resolution of $0.1 \mathrm{~s}$. Contrary to most available data which is averaged over a sea-state, a season or even a year, the CORES electrical power time series data can

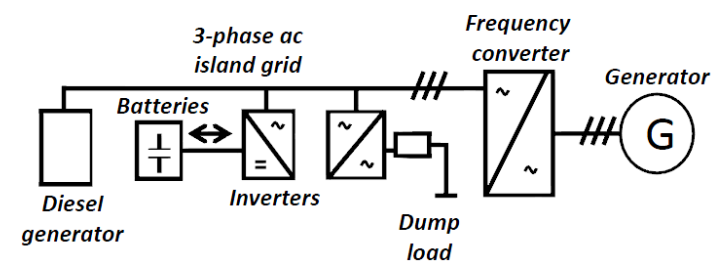

Figure 2: On-board islanded grid

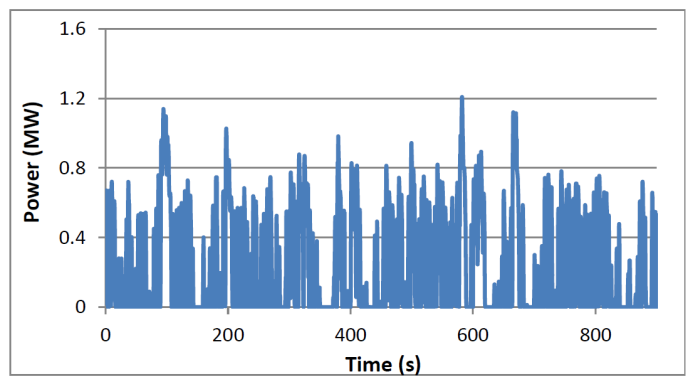

Figure 3: Power profile of an individual wave energy converter

be scaled and used directly for grid impact studies.

A power output time series was selected in order to represent a medium wave energy level typical of the west coast of Ireland. The wave climate, or sea-state, corresponding to this time series is characterized by a Pierson-Moskowitz spectrum which can be defined by a significant wave height $H_{s}$ and an energy period $T_{e}$ [9]. The significant wave height $H_{s}$ is defined as the mean wave height of the highest third of the waves while the energy $T_{e}$ is the period of an equivalent, idea sinusoidal wave whose power is equal to the sea-state power. As for the time series considered, the significant wave height $H_{s}$ is equal to $5.0 \mathrm{~m}$ and the energy period $T_{e}$ to $10.9 \mathrm{~s}$. A worst case scenario with respect to power quality was considered in this study: during this production period, the generator was operated in constant speed control mode in which, unlike in variable speed operation, inertial energy storage by means of speed control is not available. As a result, mechanical power peaks are converted directly into electrical power peaks. Fig. 3 shows the equivalent electrical power time series which would be output by a full-scale prototype for the production period considered.

Choosing this worst case scenario was motivated by the fact that the frequency range of the voltage fluctuations induced by wave farms is expected to be relatively lower than in the case of wind farms, thus corresponding to a lower level of perceptibility, as illustrated in Fig. 1. Hence, the objective of this article was to assess, by means of worst case conditions, up to which grid strength level (in terms 


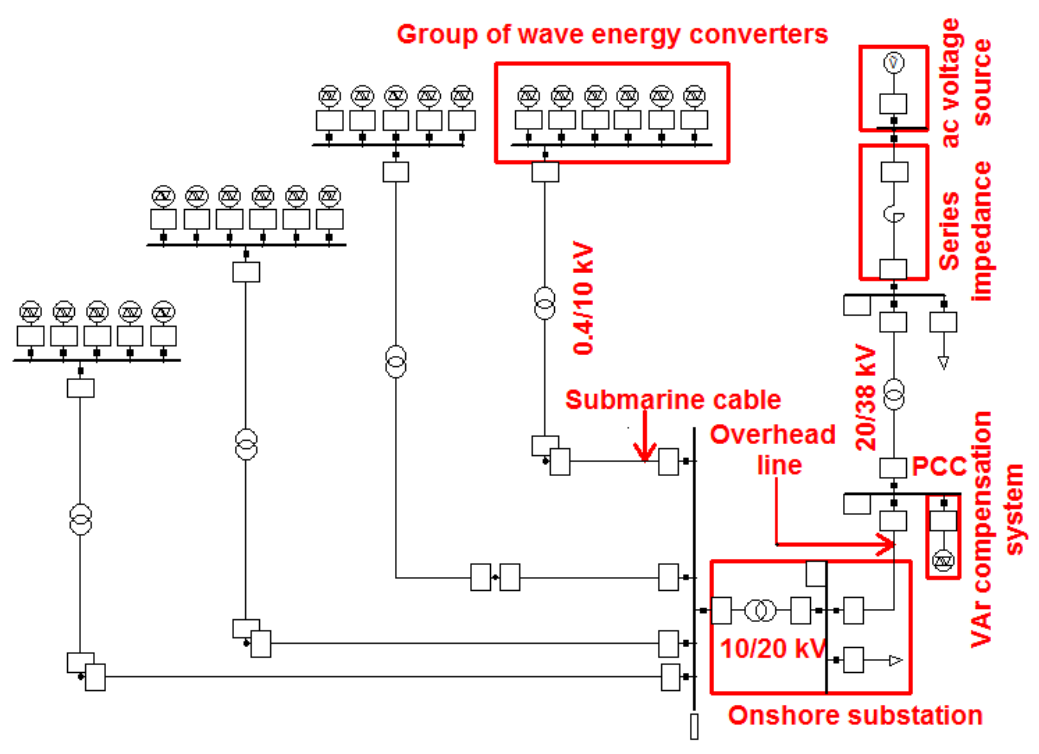

Figure 4: Schematic overview of the numerical grid model developed in PowerFactory

of short-circuit ratio and impedance angle $\Psi_{k}$ ) the grid connection of a medium-size wave farm may pose a problem in terms of flicker level. In addition, it must be noted that, as the electrical power output of a wave energy converter is highly coupled to the input wave power in fixed-speed mode, the results of the study presented in this article are representative of the maximum flicker level which can be emitted by most oscillating wave energy converters independently of their specific control strategies. It is important to stress that the analysis presented in this article constitutes the first step of a general study on the grid impact of wave farms. Subsequent studies, whose results will be published in the near future, have of course considered variable speed operation [10] which has already proved to be very efficient in reducing the flicker level generated by wind turbines [11], [12].

\section{B. Devices aggregation}

Grouping several wave energy converters together is generally thought to be beneficial in terms of power quality, by producing a smoother farm power output [13]. In this study, the effect of device aggregation on the farm power output is modeled by means of random time delays which are applied individually to the power profile of each generator.

\section{Electrical model}

The numerical grid model of the wave farm used for the simulations is shown in Fig. 4. It is inspired from the concept design of the national wave test site of Ireland (AMETS) located off Belmullet town and it presents characteristics typical of current and planned test sites, such as the WaveHub, EMEC and bimep test sites. The farm is supplied by four submarine cables, two being $6.5 \mathrm{~km}$ long and the other two being $16 \mathrm{~km}$ long. An onshore substation, connecting the point of common coupling (PCC) to the rest of the network through a $5 \mathrm{~km}$ overhead line, steps the voltage up from $10 \mathrm{kV}$ to $20 \mathrm{kV}$. A VAr compensation system, modeled generically as a controlled source of reactive power and located at the PCC, maintains power factor at this node at a fixed value, which is equal to unity in the base case. Simulations were also performed with different power factor values to study the influence of this control method on flicker level. A series reactor, whose impedance was varied to simulate different values for the short-circuit ratio and for the impedance angle $\Psi_{k}$, connected in series with an ac voltage source at $38 \mathrm{kV}$, represents the rest of the national/regional network.

\section{Simulation SCENARIOS}

The wave farm consists of 22 individual generators, leading to an equivalent full-scale rated power capacity of $19.4 \mathrm{MW}$. The generators are modeled as controlled current sources, thus simulating different generator types (synchronous generator, either with permanent magnets or brushes, or induction generator) connected to the network through a fully-rated power electronics interface. This configuration, as opposed to that corresponding to a DFIG, is very popular among wave energy developers according to an extensive survey [14]. It is important to note that the results of this study 


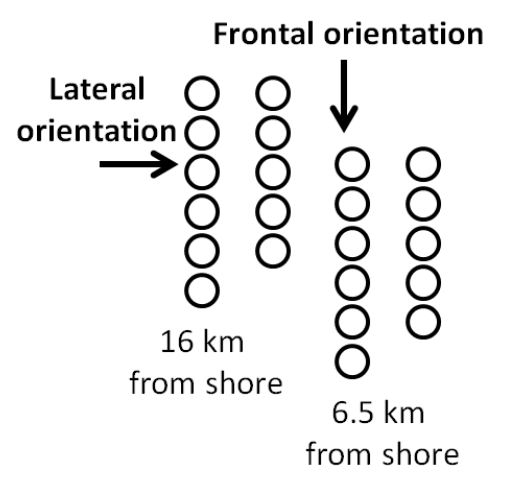

Figure 5: Orientations used in the simulations

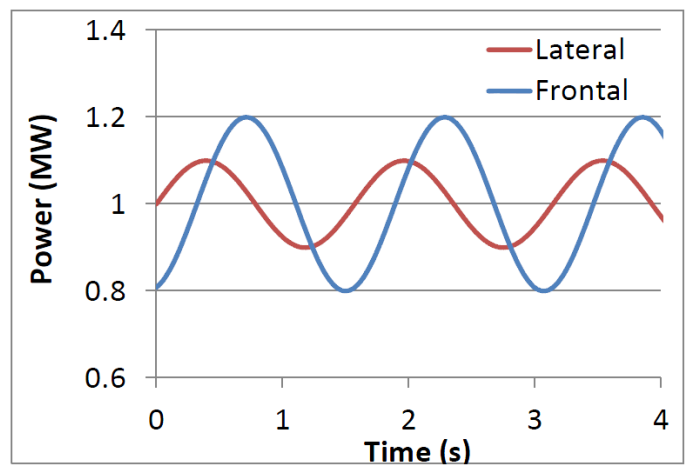

Figure 6: Power profiles of a wave farm consisting of three devices using both farm orientations

are applicable to any type of generator connected through this interface. Two orientations for the wave farm were selected, as shown in Fig. 5. In the so-called "lateral" orientation, a greater number of generators may output power peaks simultaneously (being applied the same time delay) than in the "frontal" orientation, leading to a greater impact on power quality in the former case, as the amplitude of the farm's power fluctuations is expected to be higher. For the sake of illustration, this effect was represented by means of a simple example in which three generators output a simplified, sinusoidal power profile. Fig. 6 shows the sum of these power profiles when either the frontal or the lateral orientation is used. In the first case, none of the generator power profiles is in phase with the others, leading to a smoother power output than with the lateral orientation where they all are. Simulations were performed for short-circuit ratios ranging between 3 and 100. In similar fashion to wind turbines, the power quality of the wave farm was assessed for the four following values for the impedance angle $\Psi_{k}: 30^{\circ}, 50^{\circ}, 70^{\circ}$ and $85^{\circ}$, as recommended by IEC standard 61400-21.

As mentioned previously, compliance tests with respect to flicker requirements were performed specifically for five different test sites whose char-

\begin{tabular}{|c|c|c|c|}
\hline Test site & Country & $S_{S C} / S_{\text {farm }}$ & $\Psi_{k}\left(^{\circ}\right)$ \\
\hline Achill Island & Ireland & 2.9 & 67 \\
Belmullet & Ireland & 3.2 & 69 \\
Killard & Ireland & 8.2 & 81 \\
WaveHub & UK & 32.3 & 78 \\
bimep & Spain & 232.0 & 90 \\
\hline
\end{tabular}

Table I: Actual or estimated short-circuit characteristics at the terminals of several test sites.

acteristics are presented in Table I. These sites are either in operation or at a design stage and their short-circuit ratios are representative of the entire spectrum to be expected for potential connection points. All of these test sites are currently designed, or planned to be, for a maximum power capacity of $20 \mathrm{MW}$. With the exception of the bimep test site whose characteristics were provided by courtesy of the Basque Energy Agency, Ente Vasco de la Energia (EVE), the short-circuit level and impedance angle at the other sites were estimated. This estimation was based on the shortcircuit level at the closest $110 \mathrm{kV}$ [15] or $400 \mathrm{kV}$ [16] connection point for the Irish test sites and the WaveHub respectively, and on the equivalent impedance between this connection point and the closest $38 \mathrm{kV}$ (Ireland) or $33 \mathrm{kV}$ (UK) distribution level connection point. Impedances at the WaveHub and bimep terminals were converted into equivalent impedances at $38 \mathrm{kV}$, the voltage level used in the network model. It must be noted that, due to similarities in terms of power system architecture and typical grid strength level between the rural areas of Ireland and of Scotland, the results obtained for Belmullet and Achill Island are expected to be relatively similar to those of potential sites located in this latter region. It is interesting to highlight this parallel as these two regions present the highest wave energy potential in Europe.

\section{FLICKER REQUIREMENTS}

Power plant managers must prove that their power plants comply with a number of power quality requirements, among them flicker level requirements, in order to be allowed grid connection, as these requirements ensure that power systems are operated in a safe and reliable way. Flicker is a phenomenon caused by voltage variations on lighting equipment, which results in a varying light intensity. This may represent significant disturbance to electricity customers and may even provoke epileptic seizures to persons prone to this disease [17]. In addition, sufficiently high and repetitive voltage variations were demonstrated to deteriorate the correct operation and control of electronic equipment [18]. A survey of several grid codes, recommendations and standards was conducted in 


\begin{tabular}{|c|c|c|}
\hline & Region/country & Limit \\
\hline Distribution code [19] & Ireland & $0.35^{1}$ \\
\hline Grid code [20] & Great Britain & $1.0^{2}$ \\
\hline IEC standard [21] & N/A & $\begin{array}{c}\geq 0.35^{1} \\
0.8^{2}\end{array}$ \\
\hline Nordic Grid Code [22] & $\begin{array}{c}\text { Norway, } \\
\text { Sweden, } \\
\text { Finland, East } \\
\text { Denmark }\end{array}$ & $1.0^{2}$ \\
\hline
\end{tabular}

${ }^{1}$ Individual flicker contribution of a power plant

${ }^{2}$ Total flicker level at the PCC

Table II: Short-term flicker level limits

order to determine the most stringent as well as the most permissive limits currently enforced in terms of short-term flicker level, Pst (evaluated over 10 minutes). Table II shows the limits in terms of maximum allowed flicker level as enforced by different grid operators or as recommended by an IEC standard. These limits can be expressed either as the maximum individual contribution of a single power plant to the total flicker level at the PCC, or as the total flicker level at this node, as both criteria are used.

A wave farm can be considered as compliant regardless of the pre-connection flicker level if the flicker level it induces at the PCC remains below 0.35 . This value constitutes the minimum limit which grid operators should enforce according to IEC standard 61000-3-7, and which is currently applied in Ireland for distribution networks. If on the contrary the flicker level ranges between 0.35 and unity, the farm's compliance depends on the pre-connection flicker level or on the flicker emission limit assigned to the wave farm, if any (as defined in IEC standard 61000-3-7). This limit is determined based on the percentage which the wave farm apparent power represents compared to the power capacity already installed at the PCC. Complying with flicker requirements is thus strictly site-dependent in this case. However, if flicker level exceeds 1.0, the wave farm can be considered as failing definitely to comply with the flicker requirements.

\section{FLICKERMETER DESIGN}

A flickermeter was built in Matlab according to the design specifications of IEC standards 610004-15 and 61400-21 [23]. This tool consists of five functional blocks. Block 1 per-unitizes the input voltage time series, Block 2 simulates the response in light intensity of an incandescent light bulb to voltage fluctuations, while Blocks 3 to 4 simulate the response of an average individual to light intensity variations. Finally, Block 5 performs a statistical analysis of flicker perceptibility over $10 \mathrm{~min}$.

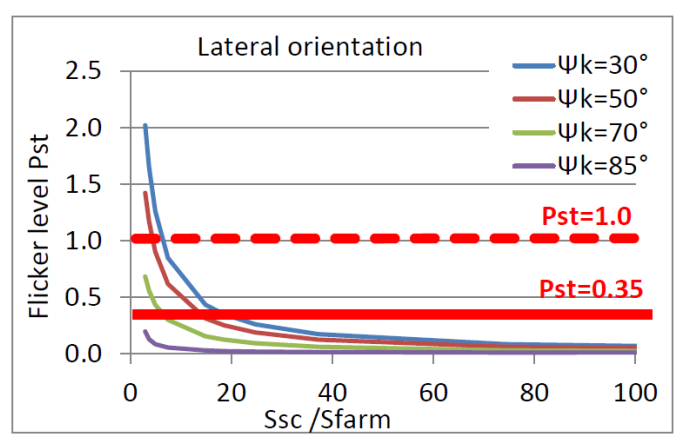

Figure 7: Flicker level versus short-circuit ratio

A high level of accuracy to the different performance tests indicated in IEC standard 61000-4-15 was demonstrated by the flickermeter designed as part of this work. Instantaneous flicker perceptibility equal to unity (within $\pm 1 \%$ ) was obtained by varying the indicative voltage amplitudes by $-3 \%$ to $+2 \%$, thus well within the maximum allowed range of $\pm 5 \%$. Similar results were obtained for the tests focusing on Block 5, short-term flicker level being obtained within $-1 \%$ to $+3 \%$ of unity, thus also well within the maximum allowed range of $\pm 5 \%$.

\section{RESUlTS: COMPLIANCE WITH FLICKER LEVEL REQUIREMENTS}

Flicker level at the PCC can reach very high values under worst case conditions, specifically for the lateral wave farm orientation, as shown in Fig. 7. The minimum short-circuit ratio recommended for preventing flicker from exceeding unity is equal to 6 and 4 for connection points whose impedance angle $\Psi_{k}$ is equal to $30^{\circ}$ to $50^{\circ}$ respectively. Nodes with a greater impedance angle are likely not to be affected by this issue, considering their typically high short-circuit ratio. As expected, the flicker level increases inversely to the impedance angle $\Psi_{k}$ which is typical of operation at unity power factor. Flicker level being highly dependent on the amplitude of the voltage variations, this trend can be easily explained by the fact that the influence of active power fluctuations on the voltage at the PCC decreases as a function of the impedance angle $\Psi_{k}$ at this node. This phenomenon is similar to what may be observed in a simple, 2-bus system for which the voltage variation $\Delta V$ between the two buses can be expressed as [24]:

$$
\Delta V=\frac{P R+Q X}{V} \approx \frac{P R}{V} \text { when } P \gg Q
$$

where $P$ and $Q$ are the active and reactive powers flowing through the series impedance $R+j X$ connecting the two nodes, and $V$ is the voltage at 
one of the nodes. All connection points, with the exception of $85^{\circ}$ points, can be affected by flicker exceeding the most stringent limit equal to 0.35 . The minimum recommended short-circuit ratio is equal to 17 and 13 for $30^{\circ}$ and $50^{\circ}$ respectively, falling down to 6 for $70^{\circ}$ points. These results seem reasonable compared to the recommended shortcircuit ratio limit used for wind farm connections. It was generally recommended to connect wind farms operating at unity power factor to connection points whose short-circuit ratio is at least equal to 25 [25], or to provide suitable power factor control if the wind farm is to be connected at nodes with a smaller short-circuit ratio [26], [27]. This strategy helps reducing the amplitude of voltage variations induced by the injection of fluctuating power, thus reducing flicker as well. These recommendations lead to flicker level not exceeding unity. As indicated in this work, flicker from wave farms is maintained below this limit, even at the lowest impedance angles, from a short-circuit ratio equal to 6 for a power factor maintained at unity. This demonstrates that the lower perceptibility of waveinduced voltage fluctuations has a greater influence than their higher amplitude compared to the case of wind farms.

Flicker at the PCC can be partially mitigated by power factor control, as expected from the experience gained from the wind energy industry [25]. Fig. 8 presents the flicker levels obtained at a connection point presenting the lowest short-circuit ratio investigated in this study and equal to 3 . The following power factor values were investigated: unity, 0.95 and 0.92 lagging (referring here as absorbing reactive power). Results show that flicker level at $50^{\circ}$ points can be reduced down to 0.36 , which is extremely close to the most stringent limit (Pst=0.35). However, as this type of control can induce a significant flicker level rise in highly reactive networks (i.e. with a high impedance angle $\Psi_{k}$ ), it is not recommended in this case. In addition, it must be stressed that complementary flicker mitigation means, such as storage and/or variable speed control mode, should be used when a farm is connected to highly resistive networks (i.e. low impedance angle $\Psi_{k}$ ) as power factor control is not sufficient in this case.

Regarding the different test sites, both Belmullet and Achill Island can be considered as failing to comply with the most stringent limit, equal to the limit enforced in Ireland, power factor control being insufficient in this case. However, wave farms connected to similarly weak connection points in countries or regions where a more permissive limit is enforced may be compliant provided that the pre-

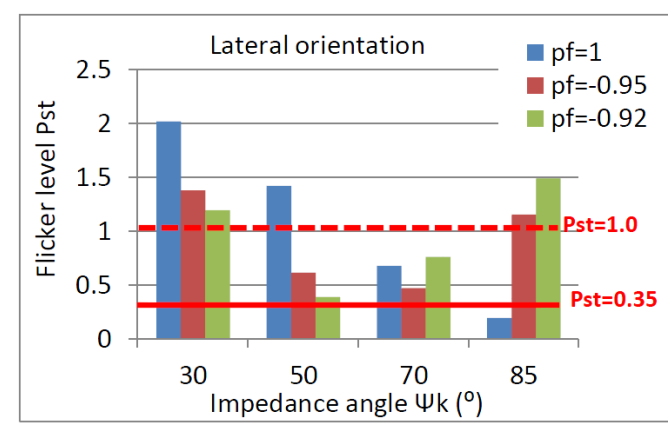

Figure 8: Flicker level for different power factors

connection flicker level remains sufficiently low. However, evaluating the total flicker level taking into account both the background flicker and the wave farm's contribution may be relatively difficult. Flicker being a non-linear phenomenon, the total flicker level is not equal to the algebraic addition of the different flicker contributions by which it is generated. Hence, precise data on the background voltage fluctuations at potential connection points would be necessary to evaluate the total flicker level, focusing in particular on the fluctuations induced by loads switching which represents the major source of flicker in distribution networks. This should be provided for instance in the form of voltage time series recorded prior to the wave farm connection, or in the form of a voltage spectrum. However, this type of data is usually not available, which prevents definitive conclusions to be drawn when the flicker level lies between 0.35 and unity.

\section{A. Analysis}

In conclusion, this study shows that wave farms without further mitigation means than power factor control at the point of common coupling are not recommended for connection to nodes whose impedance angle is less than or equal to $30^{\circ}$. This recommendation is based on power quality considerations, but seems doubly justified by the expected power system congestion issues due to the typically low power transfer capacity available on low voltage distribution networks to which these nodes usually belong. Connection points with an impedance angle of $50^{\circ}$ are also affected by this issue, which can however be mitigated to a certain extent by applying a lagging power factor. This technique may in many cases enable the wave farm to become compliant with the flicker requirements, at least the most permissive. However, if the most stringent flicker limit is enforced, additional mitigation means such as storage or variable speed mode may be required, as it may also be the case for farms connected to $70^{\circ}$ connection points. 
Connection points whose impedance angle is equal to $85^{\circ}$ are not affected by this issue.

More generally, the results imply that:

1- Medium-size wave farms can be safely connected to grids with a relatively low impedance angle $\Psi_{k}$ (down to $50^{\circ}$ ), provided the power transfer capacity is sufficient and that suitable power factor control is applied. The possible utilization of $50^{\circ}$ connection points is very interesting for regions or countries having relatively weak power systems (i.e. presenting generally both a low shortcircuit ratio and a low impedance angle $\Psi_{k}$ ), as it may be the case in developing and emerging countries, as well as in sparsely populated areas in general. This potential utilization is also interesting for providing power to partially damaged electrical power systems, for instance in the case of natural disasters or of man-made events [28].

2- In addition, it appears that wave farm owners may not necessarily need to connect their plants to a very strong connection point (i.e. presenting either a high short-circuit ratio and/or a high impedance angle $\Psi_{k}$ ) which may be located very far inland, as it is the case in the rural areas of Ireland and Scotland. Hence, they may avoid the costly installation of a long overhead line between the test site's onshore substation and the inland connection point. From a financial point of view, this means that the expensive power systems reinforcement necessary for facilitating the large scale integration of wave farms can be postponed, from a power quality perspective, until the wave energy industry reaches a certain degree of commercial maturity. This represents a major asset for the wave energy community as it will enable the different stakeholders to gain confidence in the technology before important investments are required.

\section{CONCLUSIONS}

This paper detailed the grid impact in terms of flicker level of a medium-size wave farm connected to nodes presenting a wide range of short-circuit ratios and impedance angles $\Psi_{k}$. The power system simulations presented in this paper are based on experimental electrical power profile data which was generated by a quarter-scale OWC prototype deployed at sea during a three-month period. The flicker level generated by the farm was compared to the most permissive as well as to the most stringent limits enforced by a large number of grid operators around the world. It demonstrated that the flicker generated from wave farms may constitute an issue under certain conditions. More specifically, this study shows that this type of wave farm can be safely connected, from a power quality perspective, to nodes whose impedance angle may be as low as $50^{\circ}$, provided that suitable power factor control is applied at the PCC. However, this technique being not always sufficient, additional mitigation means, such as storage and/or variable speed mode, may be required in some cases. As the wave energy converters were operated in fixed speed mode, the results presented in this article correspond to the worst case scenario in terms of power quality. Subsequent analyses addressing the grid impact of wave energy converters operated in variable speed mode, which has proved to reduce flicker considerably in the case of wind farms, were conducted and their results will be published in the near future. The article showed as well that the minimum short-circuit ratio above which the impact on power quality is negligible is smaller in the case of wave farms compared to wind farms, due to the lower frequency range of the voltage fluctuations induced by the former.

\section{ACKNOWLEDGMENTS}

Some of the data made available to this research came from a project funded by the European Community (CORES). The work of Anne Blavette and Dara O'Sullivan was supported by the Charles Parsons Award from Science Foundation Ireland. The authors wish to thank Maider Santos (Tecnalia, Spain) and the Basque Energy Agency (EVE), for providing information on the short-circuit level and impedance angle of the bimep test site. They also wish to thank Brendan Cahill (HMRC), for providing data on the sea climate at the Belmullet test site.

\section{REFERENCES}

[1] R. Alcorn, "Wave station modelling based on the Islay prototype plant", PhD thesis, Queen's University Belfast, UK, 2000.

[2] IEC 61000-4-15,“ Electromagnetic compatibility (EMC) Part 4-15: Testing and measurement techniques - Flickermeter - Functional and design specifications", TC/SC 77A, ed2.0, 2010.

[3] A. Nambiar, A. Kiprakis, D. Forehand, and A. Wallace, "Effects of array configuration, network impacts and mitigation of arrays of wave energy converters connected to weak, rural electricity networks", 2010 Proc. 3rd Int. Conf. Ocean Energy, Bilbao, Spain.

[4] A. Blavette, D L. O'Sullivan, A. W. Lewis, M. G. Egan, "Impact of a wave farm on its local grid: voltage limits, flicker level and power fluctuations", 2012 Proc. IEEE/MTS OCEANS12 Conf., Yeosu, South Korea.

[5] F. Sharkey, E. Bannon, J. MacEnri, K. Gaughan, M. Conlon, "Voltage flicker evaluation for wave energy converters assessment guidelines", 2012 Proc. 4th Int. Conf. on Ocean Energy, Dublin, Ireland.

[6] D. O’Sullivan, F. Salcedo, A. Blavette, M. Santos, A.W. Lewis, "Case studies on the benefits of energy storage for power quality enhancement: oscillating water column arrays", 2012 Proc. 4th Int. Conf. on Ocean Energy, Dublin, Ireland. 
[7] J. Aubry, "Optimisation du dimensionnement d'une chaîne de conversion électrique directe incluant un système de lissage de production par supercondensateurs. Application au houlogénérateur SEAREV", PhD thesis, Ecole Nationale Supérieure de Cachan, France, 2011.

[8] F. Thiebaut, D. O'Sullivan, P. Kracht, S. Ceballos, J. Lopez, C. Boake, J. Bard, N. Brinquete, J. Varandas, L.M.C. Gato, R. Alcorn, A.W. Lewis, "Testing of a floating OWC device with movable guide vane impulse turbine power take-off", 2011 Proc. 9th European Wave and Tidal Energy Conf., Southampton, UK.

[9] J. Falnes, Ocean Waves and Oscillating Systems: Linear Interactions Including Wave-Energy Extraction, Cambridge University Press, 2002.

[10] A. Blavette, "Grid integration of wave energy \& generic modelling of ocean devices for power system studies", $\mathrm{PhD}$ thesis, University College Cork, Ireland, 2013.

[11] A. Larsson, "Grid impact of variable-speed wind turbines", PhD thesis, Chalmers University of Technology, Göteborg, Sweden, 2000.

[12] T. Thiringer, T. Petru, and S. Lundberg, "Flicker contribution from wind turbine installations", IEEE Transactions on Energy Conversion, vol. 19, no. 1, 2004.

[13] J. Tissandier, A Babarit, A. Clément, "Study of the smoothing effect on the power production in an array of SEAREV wave energy converters", 2008 Proc. 18th Int. Offshore and Polar Engineering Conf., Vancouver, BC, Canada.

[14] D. O' Sullivan, D. Mollaghan, A.Blavette and R.Alcorn. "Dynamic characteristics of wave and tidal energy converters \& a recommended structure for development of a generic model for grid connection", a report prepared by HMRCUCC for the OES-IA Annex III. 2010. [Online], Available: www.iea-oceans.org.

[15] Transmission Forecast Statement 2011-2017, Technical report, EirGrid, 2012.

[16] National Electricity Transmission System (NETS) Seven Year Statement, British National Grid, 2011.

[17] E. Ossentjuk, C.J.O. Elink Sterk, W. Strom van Leeuwen, "Flicker-induced cardiac arrest in a patient with epilepsy", Electroencephalography and Clinical Neurophysiology, vol. 20, Issue 3, p. 257-259, 1966.

[18] M. Bollen, I. Gu (2006), Signal Processing of Power Quality Disturbances, John Wiley \& Sons.

[19] Distribution Code, version 2.0, ESB, 2007.

[20] The Grid Code, Issue 4, Revision 2, National Grid, 2010.

[21] IEC/TR 61000-3-7, "Electromagnetic compatibility (EMC) - Part 3-7: Limits - Assessment of emission limits for the connection of fluctuating installations to $\mathrm{MV}, \mathrm{HV}$ and $\mathrm{EHV}$ power systems", TC/SC 77A, ed2.0, 2008.

[22] Nordic Grid Code, NORDEL, 2007.

[23] IEC 61400-21, "Wind turbines - Part 21: Measurement and assessment of power quality characteristics of grid connected wind turbines", TC/SC 88, ed2.0, 2008.

[24] J. Glover, M. Sarma, T. Overbye (2008), Power Systems Analysis and Design, Thomson Learning.

[25] T. Ackermann, Wind Power in Power Systems, John Wiley \& Sons, 2005.

[26] S. Lundberg, "Electrical limiting factors for wind energy installations", Master thesis, Chalmers University, Sweden, 2000

[27] J. Tande, G. Di Marzio, K. Uhlen, "System Requirements for Wind Power Plants", Technical report, SINTEF, 2007.

[28] Website of the US Navy (http://www.navy.mil/), "Navy Issues New Shore Energy Policy to Achieve Energy Security Goals", published on 7/10/2012, Accessed on 24th October 2012.

Anne Blavette received her PhD from University College Cork, Ireland in 2013. She conducted her $\mathrm{PhD}$, and is still currently working at the Hydraulics \& Maritime Research Centre in Cork.
Her research interests cover the impact of ocean farms on power quality as well as the dynamic modeling of ocean energy converters for power system studies.

Dara O'Sullivan Dara L. O'Sullivan received his Ph.D. degree from University College Cork (UCC), Cork, Ireland, in 2001. He was a Senior Research Fellow at the Hydraulics and Maritime Research Centre, UCC, where he was involved in research and development in the areas of power conversion and grid integration of ocean energy technology. In 2012 he joined the Motor and Power Control group in Analog Devices International. He has published 37 papers in both international journals and conferences. He is the holder of 3 patents and was a national technical expert for Ireland in 2 IEC groups involved in standards development for ocean energy.

Ray Alcorn received his $\mathrm{PhD}$ from Queen's University Belfast, UK in 2000. He worked as the Head of Electrical Engineering at Energetech Australia Pty Ltd from 2002 to 2006. He joined the Hydraulics \& Maritime Research Centre in 2006, where he was appointed executive director in 2011.

Tony Lewis received his $\mathrm{PhD}$ from the University of Wales, Bangor in 1983. He is the academic director of the Hydraulics \& Maritime Research Centre and he is also in charge of the Chair of Energy at University College Cork.

Michael G. Egan received his $\mathrm{PhD}$ from the National University of Ireland, Dublin, Ireland, in 1985. He is a Statutory Lecturer at University College Cork and the founder, as well as the director of the Power Electronics Research Laboratory. His research interests are in the analysis and practical applications of power electronic converters. 